\title{
Tauroursodeoxycholic acid exerts anticholestatic effects by a cooperative cPKC $\alpha$-/PKA-dependent mechanism in rat liver
}

\author{
R Wimmer, ${ }^{1}$ S Hohenester, ${ }^{1}$ T Pusl, ${ }^{1}$ G U Denk, ${ }^{1}$ C Rust, ${ }^{1}$ U Beuers ${ }^{1,2}$
}

${ }^{1}$ Department of Medicine II,

Klinikum Grosshadern, University of Munich, Germany;

${ }^{2}$ Department of

Gastroenterology \& Hepatology,

Academic Medical Center,

University of Amsterdam, The

Netherlands

Correspondence to:

Professor U Beuers, Department of Gastroenterology \&

Hepatology, Academic Medical

Center, University of

Amsterdam, P0 Box 22700, NL-

1100 DE Amsterdam, The

Netherlands; u.h.beuers@

amc.uva.nl

RW and SH contributed equally to this study.

Data in this study were reported, in part, at the Annual Meeting of the European Association for the Study of the Liver, Barcelona, 13 April 2007, and at the Biannual International Bile Acid Meeting, Freiburg, 7

October 2006, and were pub-

lished in part in abstract form in

J Hepatol 2007;:46(Suppl

I):S119.

Revised 15 May 2008

Accepted 29 May 2008

Published Online First

26 June 2008

\begin{abstract}
Objective: Ursodeoxycholic acid (UDCA) exerts anticholestatic effects in part by protein kinase $C$ (PKC)dependent mechanisms. Its taurine conjugate, TUDCA, is a $\mathrm{CPKC} \alpha$ agonist. We tested whether protein kinase $\mathrm{A}$ (PKA) might contribute to the anticholestatic action of TUDCA via cooperative CPKC $\alpha$-/PKA-dependent mechanisms in taurolithocholic acid (TLCA)-induced cholestasis.

Methods: In perfused rat liver, bile flow was determined gravimetrically, organic anion secretion spectrophotometrically, lactate dehydrogenase (LDH) release enzymatically, cAMP response-element binding protein (CREB) phosphorylation by immunoblotting, and CAMP by immunoassay. PKC/PKA inhibitors were tested radiochemically. In vitro phosphorylation of the conjugate export pump, Mrp2/Abcc2, was studied in rat hepatocytes and human Hep-G2 hepatoma cells.
\end{abstract}

Results: In livers treated with TLCA $(10 \mu \mathrm{mo} / /)+$ +TUDCA ( $25 \mu \mathrm{mol} / \mathrm{l})$, combined inhibition of cPKC by the cPKCselective inhibitor Gö6976 (100 nmol//) or the nonselective PKC inhibitor staurosporine $(10 \mathrm{nmol} / \mathrm{l})$ and of PKA by H89 (100 nmol//) reduced bile flow by $36 \%$ $(p<0.05)$ and $48 \%(p<0.01)$, and secretion of the Mrp2/ Abcc2 substrate, 2,4-dinitrophenyl-S-glutathione, by $31 \%$ $(p<0.05)$ and $41 \%(p<0.01)$, respectively; bile flow was unaffected in control livers or livers treated with TUDCA only or TLCA+taurocholic acid. Inhibition of CPKC or PKA alone did not affect the anticholestatic action of TUDCA. Hepatic CAMP levels and CREB phosphorylation as readout of PKA activity were unaffected by the bile acids tested, suggesting a permissive effect of PKA for the anticholestatic action of TUDCA. Rat and human hepatocellular Mrp2 were phosphorylated by phorbol ester pretreatment and recombinant ${ }_{\mathrm{CPKC}} \alpha, \mathrm{nPKC} \varepsilon$, and PKA, respectively, in a staurosporine-sensitive manner.

Conclusion: UDCA conjugates exert their anticholestatic action in bile acid-induced cholestasis in part via cooperative post-translational $c P K C \alpha-/ P K A$-dependent mechanisms. Hepatocellular Mrp2 may be one target of bile acid-induced kinase activation.

Ursodeoxycholic acid (UDCA) has been used for the treatment of jaundice in Chinese traditional medicine since the Tang dynasty (618-907 AD) in the form of dried black bear's bile. Today, UDCA represents the only drug approved by the US Food and Drug Administration for the treatment of primary biliary cirrhosis (PBC), a model cholestatic liver disease. ${ }^{1}$ UDCA improves biliary secretion in $\mathrm{PBC}$ and a number of other cholestatic disorders, such as primary sclerosing cholangitis (PSC) or intrahepatic cholestasis of pregnancy (ICP). ${ }^{2}$ In early-stage $\mathrm{PBC}$, UDCA delays progression to cirrhosis $^{3}$ as well as development of complications, ${ }^{5}$ and normalises life expectancy. ${ }^{6-8}$ Several mechanisms of action of UDCA have been discussed, and stimulation of impaired hepatobiliary secretion, detoxification of bile, and antiapoptotic effects are assumed to contribute to the beneficial effect of UDCA in cholestatic disorders. ${ }^{9}$

UDCA conjugates, such as tauroursodeoxycholic acid (TUDCA), are potent signalling molecules both in hepatocytes and cholangiocytes. ${ }^{910}$ In hepatocytes, TUDCA has been shown to induce increases of cytosolic free calcium $\left[\mathrm{Ca}^{2+}\right]_{\mathrm{i}}$ and $\mathrm{Ca}^{2+}$ influx, ${ }^{11-13}$ to selectively activate $\mathrm{Ca}^{2+}$-dependent conventional protein kinase $\mathrm{C} \alpha(\mathrm{cPKC} \alpha){ }^{14}{ }^{15}$ to stimulate an integrin-dependent dual signalling pathway leading to activation of mitogen-activated protein kinases (MAPKs), Erk1/2 and p38MAPK ${ }^{16-19}$ and to induce targeting and insertion of key apical transporters like the bile salt export pump, Bsep/Abcb11, and the conjugate export pump, Mrp2/Abcc2, into canalicular membranes of hepatocytes. ${ }^{17} 2021$ In normal hepatocytes, MAPK-dependent mechanisms mediate, in part, the choleretic effect of TUDCA, ${ }^{16}{ }^{17}$ whereas in experimental cholestasis, PKC-dependent mechanisms appear to contribute to the anticholestatic action of TUDCA. ${ }^{20} 22$

Taurolithocholic acid (TLCA) is the most potent cholestatic agent among the major human bile acids $^{23}$ and has recently been shown to exert its cholestatic action at the hepatocyte level by phosphatidylinositol-3-kinase, and putatively nPKC $\varepsilon$-dependent mechanisms in isolated perfused rat livers (IPRLs) and isolated rat hepatocyte couplets. ${ }^{24}$ Like TUDCA, TLCA is a potent signalling molecule which elevates hepatocellular $\left[\mathrm{Ca}^{2+}\right]_{i}$ without stimulation of $\mathrm{Ca}^{2+}$ influx, ${ }^{25}$ selectively translocates $\mathrm{nPKC} \varepsilon$ to canalicular membranes and activates membrane-bound $\mathrm{PKC},{ }^{26}$ and induces retrieval of key apical transporters such as the bile salt export pump, Bsep/Abcb11, from canalicular membranes of hepatocytes. ${ }^{21}$

Direct effects of bile acids such as TLCA or TUDCA on PKA activity in hepatocytes have not been disclosed although glucagon-induced cAMP formation was impaired by TUDCA in a staurosporine-sensitive fashion in hamster hepatocytes. ${ }^{27}$ PKC agonists at moderate concentrations and PKA are known to stimulate liver cell secretion..$^{10} \mathrm{~A}$ cooperative PKC-/PKA-dependent mechanism has recently been described to potentiate chloride secretion via the Xenopus cystic fibrosis transmembrane conductance regulator, XCFTR ${ }^{28}$ in Xenopus oocytes, and concomitant activation of $\mathrm{cPKC} \alpha$ and PKA led to marked stimulation of insulin secretion 
Table 1 Effect of the protein kinase C (PKC) inhibitors staurosporine (ST, $10 \mathrm{nmol} / \mathrm{l})$ and Gö6976 (100 nmol/l), and the PKA inhibitor $\mathrm{H} 89$ (100 nmol/l) on activity of recombinant $\mathrm{cPKC} \alpha, \mathrm{nPKC} \varepsilon$ and $\mathrm{PKA}$ in vitro using myelin basic protein as kinase substrate

\begin{tabular}{|c|c|c|c|c|c|c|}
\hline \multirow[b]{2}{*}{ Inhibitor } & \multicolumn{2}{|l|}{ сPKC $\alpha$} & \multicolumn{2}{|l|}{ nPKC $\varepsilon$} & \multicolumn{2}{|l|}{ PKA } \\
\hline & $\begin{array}{l}\text { Concentration } \\
\text { (nmol/min/g) }\end{array}$ & $\%$ & $\begin{array}{l}\text { Concentration } \\
\text { (nmol/min/g) }\end{array}$ & $\%$ & $\begin{array}{l}\text { Concentration } \\
(\mathrm{pmol} / \mathrm{min} / \mathrm{mU})\end{array}$ & $\%$ \\
\hline $\mathrm{H}_{2} \mathrm{O}$ standard & $309(74)$ & 100 & 77 & 100 & $4.4(1.7)$ & 100 \\
\hline DMSO control & $294(46)$ & 96 & $71(11)$ & 96 & $4.7(1.6)$ & 111 \\
\hline ST & $23(3)^{* *}$ & 8 & $7(1)^{* *}$ & 9 & $1.0(0.6)^{*}$ & 22 \\
\hline Gö6976 & $163(52)$ & 53 & $61(10)$ & 82 & $4.2(1.5)$ & 98 \\
\hline H89 & $217(53)$ & 71 & $44(8)$ & 58 & $0.2(0.1)^{* *}$ & 5 \\
\hline $\mathrm{ST}+\mathrm{H} 89$ & $23(4)^{* *}$ & 8 & $6(1)^{* *}$ & 8 & $0.1(0.1)^{* *}$ & 2 \\
\hline Gö6976+H89 & $107(20)^{* *}$ & 35 & $44(5)$ & 60 & $0.4(0.2)^{* *}$ & 10 \\
\hline
\end{tabular}

PKC and PKA inhibitors were tested at concentrations at least 5- to 10 -fold above their elsewhere reported IC $\mathrm{C}_{50}$ for $\mathrm{CPKC} \alpha$ and $\mathrm{PKA}$ respectively, at which they did not affect bile flow and organic anion secretion in isolated perfused rat livers. For details see Materials and methods. Results are given as mean (SD) of protein kinase activity of four independent experiments $\left(\%\right.$ of $\mathrm{H}_{2} \mathrm{O}$ standard).

${ }^{*} p<0.05,{ }^{* *} p<0.01$ vs DMSO control $(0.1 \%, v / v)$, ANOVA post hoc test (Tukey).

DMSO, dimethylsulfoxide.

in pancreatic beta cells by a convergent mechanism. ${ }^{29}$ Therefore, we tested the hypothesis that TUDCA may exert anticholestatic effects in the well-established model of TLCA-induced cholestasis by a cooperative $c P K C \alpha$-/PKA-dependent mechanism.

\section{MATERIALS AND METHODS Materials}

Bile acids and dimethylsulfoxide (DMSO) were purchased from Sigma (St. Louis, Missouri, USA). 1-Chloro-2,4-dinitrobenzene (CDNB) was from ICN Biomedicals (Aurora, Ohio, USA). The nonselective PKC inhibitor stauroporine (ST), the cPKC inhibitor Gö6976, the PKA inhibitor H89, and the recombinant catalytic subunits of $\mathrm{cPKC} \alpha, \mathrm{nPKC} \varepsilon$ and PKA were from CalbiochemNovabiochem (Nottingham, UK). The anti-MRP2/ABCC2 antibody was from Alexis (Lausen, Switzerland). A rabbit anti-pCREB antibody and a monoclonal CREB antibody were from Cell Signalling (Danvers, Massachusetts, USA), and a monoclonal mouse anti-GAPDH antibody was from Abcam (Cambridge, UK). A goatanti-rabbit-IgG-HRP-conjugate-antibody was from Bio-Rad Lab (Munich, Germany) and a goat-anti-mouse-IgG-HRP antibody was from Santa Cruz Biotechnology (Santa Cruz, California, USA). Complete protease inhibitor cocktail was from Roche Diagnostics (Mannheim, Germany). Marker molecular weight standard was from Santa Cruz Biotechnology. The Renaissance western blot chemiluminescence reagent was from NEN (Boston, Massachusetts, USA). Hyperfilm ECL was from Amersham (Little Chalfont, UK). Polyvinylidene difluoride membranes were from Millipore (Bedford, Massachusetts, USA). A cAMP enzyme immunoassay kit was from Amersham Biosciences (Freiburg, Germany). FosCholin-12 was from Anatrace (Maumee, Ohio, USA). Protein A-Sepharose was from Zymed (San Francisco, California, USA). All other chemicals were of the highest purity commercially available.

\section{Protein kinase inhibitors}

The effect of inhibitors on activity of $\mathrm{CPKC} \alpha, \mathrm{nPKC} \varepsilon$, and PKA was tested in vitro at levels at least 5 - to 10 -fold above their IC $_{50}$ for $\mathrm{CPKC} \alpha$, as indicated in the literature, using myelin basic protein (MBP) as substrate to apply concentrations in the IPRLs high enough to be effective, but not too high to cause cholestatic effects. The results showed that $\mathrm{cPKC} \alpha$ was more effectively blocked by the nonselective PKC inhibitor staurosporine $(10 \mathrm{nmol} / \mathrm{l})$ than by the cPKC-specific inhibitor Gö6976 (10-100 nmol/l) (table 1). nPKC $\varepsilon$ was inhibited by staurosporine $(10 \mathrm{nmol} / \mathrm{l})$, but not by Gö6976 (10-100 nmol/1). In addition, the selective PKA inhibitor,
H89 (100 nmol/l), and staurosporine (10 nmol/l), but not Gö6976 blocked PKA effectively (table 1).

\section{Animals}

Male Sprague-Dawley rats (208 (SD 22) g) were obtained from Charles River (Sulzfeld, Germany) and were subjected to a $12 \mathrm{~h}$ day-night rhythm with free access to rodent chow and water.

Isolated rat liver perfusions were performed as described in detail previously. ${ }^{20}$ Bile flow was determined gravimetrically in pretared tubes.

\section{Perfusion protocol}

Rat livers were perfused for a total of $115 \mathrm{~min}$ in a nonrecirculating fashion with Krebs-Ringer bicarbonate solution $\left(\mathrm{pH} 7.4,37^{\circ} \mathrm{C}\right)$. Twenty-five minutes after starting the perfusion, the nonselective PKC inhibitor staurosporine (final concentration in the portal vein, $10 \mathrm{nmol} / \mathrm{l})$, the cPKC inhibitor Gö6976 (100 nmol/l), and/or the PKA inhibitor H89 (100 nmol/l) were infused continuously into the perfusion medium. Forty-five minutes after start of the perfusion, bile acids (TUDCA, TLCA, TCA, TUDCA+TLCA, TCA+TLCA), dibutyryl cAMP (dbcAMP) or the carrier DMSO only (control, $0.1 \%, \mathrm{v} / \mathrm{v}$ ) were added continuously to the buffer by using an infusion pump to reach

Table 2 cAMP levels and protein kinase A (PKA) activity in liver tissue are not affected by the bile acids taurolithocholic acid (TLCA) and tauroursodeoxycholic acid (TUDCA)

\begin{tabular}{llll}
\hline Bile acid & Inhibitor & cAMP (pmol/g liver) & pCREB (AE) \\
\hline DMS0 & & $30.6(3.5)$ & $0.52(0.07)$ \\
DMS0 & ST+H89 & $30.1(10.6)$ & $0.25(0.04)^{* *}$ \\
TUDCA & & $24.9(7.7)$ & $0.37(0.15)$ \\
TUDCA & ST+H89 & $29.6(7.4)$ & $0.26(0.11)$ \\
TLCA & & $30.8(8.7)$ & $0.34(0.11)$ \\
TLCA & ST+H89 & $36.6(3.3)$ & $0.22(0.07)$ \\
TLCA+TUDCA & & $31.7(6.5)$ & $0.36(0.22)$ \\
TLCA+TUDCA & ST+H89 & $39.5(14.0)$ & $0.32(0.14)$
\end{tabular}

cAMP levels and phosphorylated cAMP response-element binding protein ( $p C R E B)$ levels as a readout of PKA activity were determined with an enzyme-linked immunosorbent assay and by immunoblotting, respectively, in shock-frozen liver tissue after perfusion with the bile acids TLCA ( $10 \mu \mathrm{mol} / \mathrm{l})$ and/or TUDCA ( $25 \mu \mathrm{mol} / \mathrm{l})$ or their carrier, dimethyl sulfoxide (DMSO; $0.1 \%, \mathrm{v} / \mathrm{v}$ ) in the presence or absence of the potent PKC inhibitor staurosporine (ST, $10 \mathrm{nmol} / \mathrm{l}$ ) and the PKA inhibitor $\mathrm{H} 89$ (100 nmol//; see fig 1 and table 1). Significant reduction of pCREB, but not cAMP in the presence of $\mathrm{H} 89$ in control livers depicts the specificity of action of this PKA inhibitor. For details, see Materials and methods. Mean (SD) of four to five experiments, each.

${ }^{* *} \mathrm{p}<0.001$ vs inhibitor-free perfusion; Student $t$ test. 
final portal venous concentrations of $25 \mu \mathrm{mol} / 1$ (TUDCA, TCA) or $10 \mu \mathrm{mol} / \mathrm{l}$ (TLCA, dbcAMP). Sixty-five minutes after start of the perfusion, 1-chloro-2,4-dinitrobenzene (CDNB; $30 \mu \mathrm{mol} / \mathrm{l}$ ), the precursor of the model Mrp2/Abcc2 substrate, 2,4-dinitrophenyl-S-glutathione (GS-DNP), was infused for $10 \mathrm{~min}$. At this concentration, saturation of the biliary GS-DNP secretion was observed in the perfused rat liver..$^{20}$

\section{Biliary secretion of 2,4-dinitrophenyl-S-glutathione}

The model Mrp2/Abcc2 substrate, GS-DNP, was determined in bile spectrophotometrically as described previously. ${ }^{20}$

\section{Hepatovenous efflux of lactate dehydrogenase}

Lactate dehydrogenase (LDH) was determined as an indicator of liver cell damage in the hepatovenous effluate by use of a standard enzymatic test. ${ }^{20}$

\section{cAMP in liver tissue}

cAMP was extracted from liver tissue with a liquid phase extraction method conforming to the supplier's protocol and was determined by an enzyme immunoassay.

\section{pCREB in liver tissue}

An aliquot of shock-frozen liver tissue $\left(-80^{\circ} \mathrm{C}\right)$ was homogenised, and proteins were separated by western blotting. pCREB and GAPDH were identified by use of specific antibodies. $p C R E B$ and GAPDH were semiquantified by densitometry and pCREB was expressed as a ratio $\mathrm{pCREB} / \mathrm{GAPDH}$.

\section{Rat hepatocyte isolation and in vitro phosphorylation of Mrp2/ Abcc2}

Rat hepatocytes were isolated and cultured on collagen-coated wells as described previously. ${ }^{30}$ A sample of $5 \times 10^{6}$ cells/well was incubated for $2 \mathrm{~h}$ after plating for $4 \mathrm{~h}$ with ${ }^{32} \mathrm{P}$-orthophosphoric acid $(74 \mathrm{MBq})$ and were then treated for $30 \mathrm{~min}$ with DMSO $(0.1 \%, \mathrm{v} / \mathrm{v}), 100 \mathrm{nmol} / \mathrm{l}$ phorbol 12-myristate 13-acetate (PMA), $100 \mathrm{nmol} / 1 \mathrm{PMA}+10 \mathrm{nmol} / 1$ staurosporine, 25 mol/l TUDCA, or $10 \mu \mathrm{mol} / 1$ TLCA. Cells were then washed three times with phosphate-buffered saline (PBS).

\section{Immunoprecipitation of rat Mrp2/Abcc2 or human MRP2/ABCC2} Rat Mrp2/Abcc2 and human MRP2/ABCC2 were immunoprecipitated from rat hepatocytes and human HepG2 hepatoma cells, respectively, as published previously. ${ }^{31}$ Efficacy of Mrp2/ Abcc2 immunoprecipitation was tested by western blotting.

\section{Phosphorylation of human MRP2/ABCC2 in vitro}

Phosphorylation was peformed using a phosphorylation buffer containing $10 \mu \mathrm{mol} / 1 \mathrm{ATP}$ and per sample $370 \mathrm{kBq}\left[\gamma^{32}\right] \mathrm{ATP}$, and $400 \mathrm{U}$ activated PKA or $90 \mathrm{ng}$ activated $\mathrm{cPKC} \alpha$ or/and 110 ng activated nPKCe. MBP was used as a phosphorylation control. Samples were immunoblotted and autoradiography of the gels was performed for $18 \mathrm{~h}$. The bands corresponding to ${ }^{32} \mathrm{P}-\mathrm{MRP} 2 / \mathrm{ABCC} 2$ were excised and radioactivity was counted in a scintillation counter.

\section{Statistics}

Data are expressed as mean (SD). Results were compared between different groups using ANOVA post hoc test (Tukey). Comparison of two groups only was performed using an unpaired two-tailed Student $t$ test. A value of $p<0.05$ was considered statistically significant.

\section{RESULTS}

\section{Bile flow}

TLCA $(10 \mu \mathrm{mol} / \mathrm{l})$ reduced bile flow in isolated perfused rat livers (IPRLs) to $14 \%$ of controls (figs $1 \mathrm{~A}, \mathrm{~B}$ and $2 \mathrm{~A}$ ). TUDCA $(25 \mu \mathrm{mol} / \mathrm{l})$ reversed TLCA-induced inhibition of bile flow to $173 \%$ of controls. The nonselective PKC inhibitor staurosporine (10 nmol/l), the selective cPKC inhibitor Gö6976 (100 nmol/l), and the selective PKA inhibitor $\mathrm{H} 89$ (100 nmol/l) did not significantly affect bile flow in either control livers or in livers treated with TLCA+TUDCA (figs $1 \mathrm{~A}, \mathrm{~B}$ and $2 \mathrm{~A}$ ). In contrast, when administered concomitantly, staurosporine+H89 as well as Gö6976+H89 induced a significant reduction of bile flow in livers treated with TLCA+TUDCA by $48 \%(p<0.01)$ and $36 \%$ $(p<0.05)$, respectively, but again, did not affect bile flow in controls or livers treated with TUDCA only (figs $1 \mathrm{~A}-\mathrm{C}$ and $2 \mathrm{~A}$ ).

The cAMP analogue, dibutyryl cAMP (dbcAMP, $10 \mu \mathrm{mol} / \mathrm{l}$ ), stimulated bile flow in controls by $55 \%(p<0.01)$, but did not significantly enhance bile flow in livers treated with TLCA $(+81 \%$, NS) or TLCA+TUDCA $(+16 \%, N S)$ indicating that stimulation of PKA only does not reverse TLCA-induced cholestasis in IPRL.

The taurine conjugate of the major human trihydroxy bile acid, cholic acid (TCA, $25 \mu \mathrm{mol} / \mathrm{l}$ ) also reverses TLCA-induced inhibition of bile flow in rat liver. ${ }^{32}$ Unlike TUDCA, TCA does not affect hepatocellular $\left[\mathrm{Ca}^{2+}\right]_{\mathrm{i}}$ and $\mathrm{PKC}$ isoform distribution at physiological concentrations. ${ }^{11}{ }^{14}$ Indeed, reversal of TLCAinduced inhibition of bile flow by TCA was not affected by staurosporine+H89 (fig 2A) suggesting different molecular mechanisms mediating the anticholestatic action of TUDCA and TCA in TLCA-induced cholestasis.

Together, these data suggest that recovery of bile flow by TUDCA in TLCA-induced cholestasis - but not stimulation of bile flow under non-cholestatic conditions - is mediated by a cooperative cPKC-/PKA-dependent mechanism.

\section{Secretion of 2,4-dinitrophenyl-S-glutathione}

TLCA reduced biliary secretion of the Mrp2/Abcc2 substrate, 2,4-dinitrophenyl-S-glutathione (GS-DNP), to $7 \%$ of controls. TUDCA reversed TLCA-induced inhibition of biliary GS-DNP secretion to $113 \%$ of controls (fig $2 \mathrm{~B}$ ). The nonselective PKC inhibitor staurosporine impaired biliary GS-DNP secretion by $32 \%(p<0.05)$ and the cPKC inhibitor Gö6976 tended to impair biliary GS-DNP secretion by $24 \%$ in livers treated with TLCA+TUDCA (fig 2B), but not in control livers. The PKA inhibitor H89 did not affect biliary GS-DNP secretion in livers treated with TLCA+TUDCA (fig $2 \mathrm{~B}$ ) when given alone. When administered concomitantly, staurosporine+H89 as well as Gö6976+H89 reduced biliary GS-DNP secretion by $41 \%$ $(p<0.01)$ and $31 \%(p<0.05)$, respectively, in livers treated with TLCA + TUDCA. In contrast, biliary GS-DNP secretion in controls or livers treated with TUDCA only was barely affected by PKC/PKA inhibitors (fig 2B). Biliary GS-DNP secretion was not further enhanced by the cAMP analogue, dbcAMP, in controls, livers treated with TLCA or livers treated with TLCA+TUDCA $(-5.7 \%,+1.6 \%$, and $-49 \%$, respectively). Together, these data indicate that TUDCA-induced recovery of organic anion secretion via Mrp2/Abcc2 is mediated by a cooperative cPKC-/PKA-dependent mechanism in TLCAinduced cholestasis.

\section{Hepatic cAMP levels and PKA activity}

Hepatic cAMP levels were unaffected by any of the bile acids and inhibitors of PKC and PKA tested (table 2). In addition, CREB phosphorylation as a readout of PKA activity in liver 
Figure 1 Bile flow is stimulated by tauroursodeoxycholic acid (TUDCA) via a cooperative $c$ protein kinase $\mathrm{C} /$ protein kinase $\mathrm{A}$ (cPKC-/PKA)-dependent mechanism in taurolithocholic acid (TLCA)-induced cholestasis, but not under control conditions. (A) Bile flow was markedly impaired by TLCA $(10 \mu \mathrm{mol} / \mathrm{l})$ in isolated perfused rat livers. TUDCA (25 $\mu \mathrm{mol} / \mathrm{l})$ reversed TLCA-induced cholestasis. The nonselective PKC antagonist staurosporine $(10 \mathrm{nmol} / \mathrm{l})$, but not the selective PKA inhibitor H89 (100 nmol/l), when given alone, tended to impair the anticholestatic effect of TUDCA whereas combined administration of staurosporine and the selective PKA inhibitor H89 (100 nmol/l) significantly impaired the anticholestatic effect of TUDCA. (B) The selective cPKC inhibitor Gö6976 (100 nmol/l), when given alone, did not impair the anticholestatic effect of TUDCA in TLCA-induced cholestasis. Combined administration of Gö6976 and H89 led to a significant impairment of the anticholestatic effect of TUDCA in TLCAinduced cholestasis. (C) Combined administration of the nonselective PKC inhibitor staurosporine $(10 \mathrm{nmol} / \mathrm{l})$ and the PKA inhibitor, $\mathrm{H} 89(100 \mathrm{nmol} / \mathrm{l})$, did neither affect bile flow of DMSO controls nor of livers treated with TUDCA or TLCA alone (see also fig 2). All results are given as means (SD) of four to five experiments, each. For statistical evaluation, see fig 2. Results for TLCA in panels $B$ and $C$, and for TLCA + TUDCA in panel $B$ are identical to those in panel $A$ and are provided for clarity.
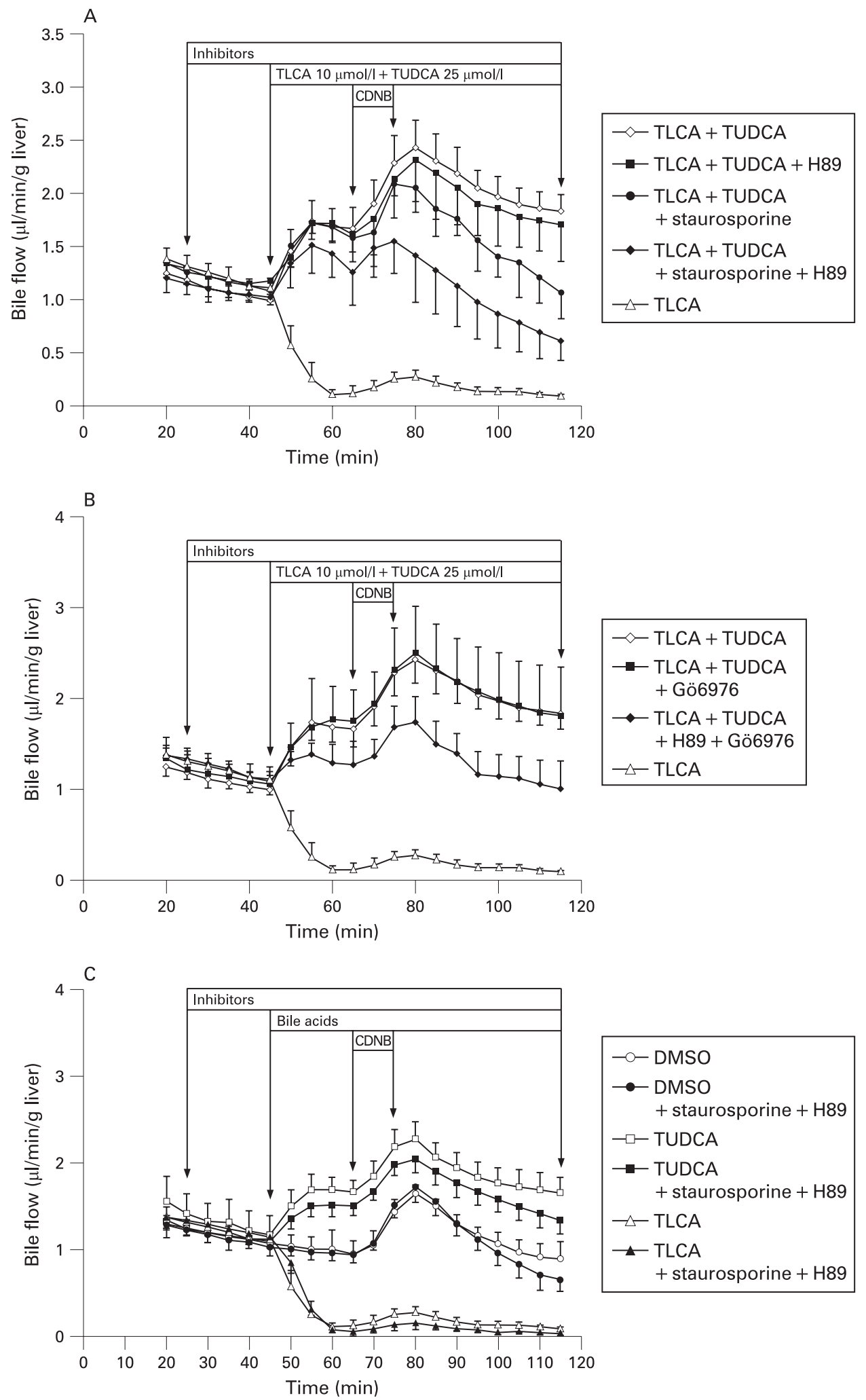

tissue was unaffected by the bile acids used (table 2) suggesting that PKA activity, in line with previous findings, ${ }^{10}{ }^{27}$ is rather permissive for the anticholestatic action of TUDCA.

\section{Hepatovenous LDH release}

The cholestatic effect of TLCA is associated with a serious cytotoxic action as demonstrated by a 32 -fold increase of hepatovenous $\mathrm{LDH}$ release as a readout of liver cell damage. TLCA-induced $\mathrm{LDH}$ release was attenuated by addition of TUDCA, but markedly increased after combined inhibition of PKC and PKA in livers treated with TLCA+TUDCA - but not after inhibition of either PKC or PKA alone - in parallel with the inhibition of the anticholestatic action of TUDCA (table 3). In contrast, $\mathrm{LDH}$ release was unaffected by combined inhibition of $\mathrm{PKC}$ and PKA in controls and livers treated with TUDCA or 

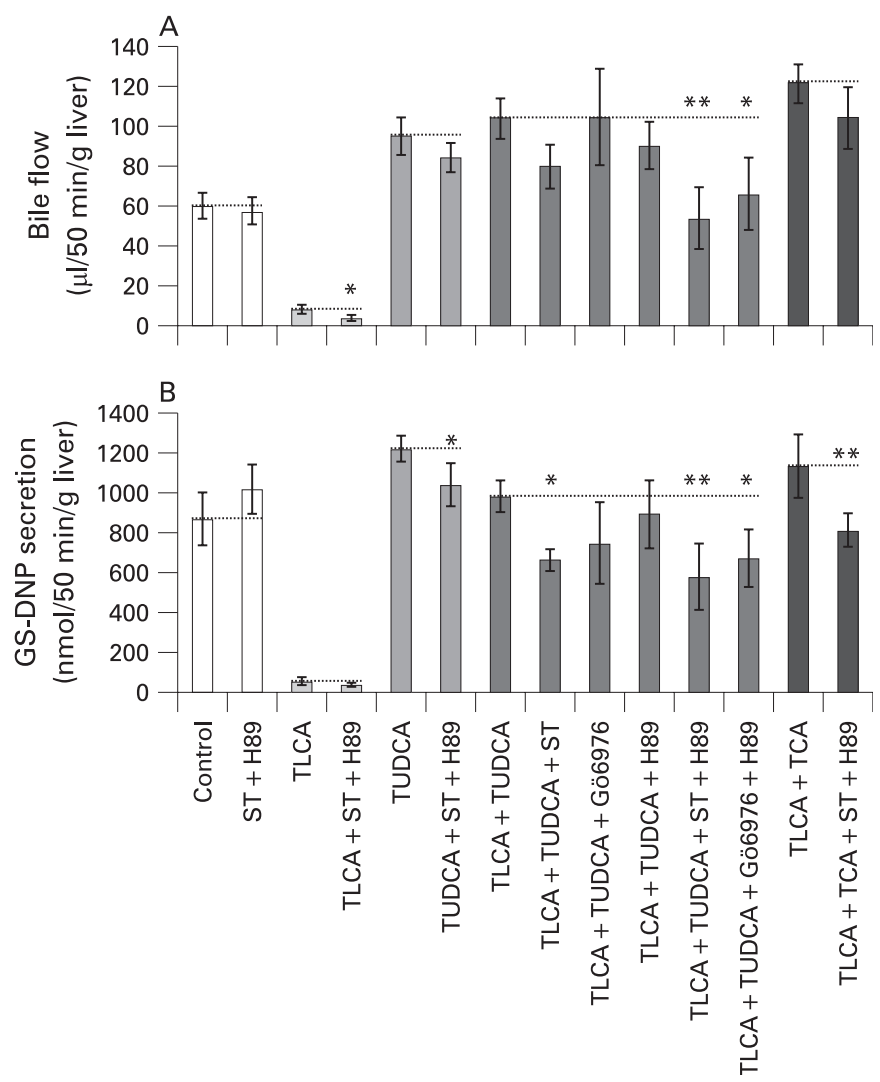

Figure 2 Bile flow and organic anion secretion are stimulated by tauroursodeoxycholic acid (TUDCA) via a cooperative $c$ protein kinase C-/protein kinase A (cPKC-/PKA)-dependent mechanism in taurolithocholic acid (TLCA)-induced cholestasis. (A) Total bile flow during the last 50 min after addition of inhibitors, the bile acids, TLCA, TUDCA and cholic acid (TCA), and the 2,4-dinitro-S-glutathione (GS-DNP) precursor, 1-chloro-2,4-dinitrobenzene (CDNB), for the experiments shown in fig $1 A, B$. (B) Biliary GS-DNP secretion during the last 50 min after addition of inhibitors, the bile acids, TLCA, TUDCA and TCA, and the GS-DNP precursor CDNB. All results are given as means (SD) of four to five experiments, each. ${ }^{*} p<0.05,{ }^{* *} p<0.01$, ANOVA post hoc test.

TLCA alone (table 3). These data suggest that TUDCA alleviates TLCA-induced liver cell damage in part by a cooperative cPKC $\alpha$-/PKA-dependent mechanism in association with its anticholestatic action.

\section{Phosphorylation of rat Mrp2 by the nonselective PKC agonist, phorbol 12-myristate 13-acetate}

Rat Mrp2 was immunoprecipitated from freshly isolated rat hepatocytes in short-term culture after incubation of hepatocytes with ${ }^{32} \mathrm{P}$-orthophosphoric acid for $4 \mathrm{~h}$ and with DMSO (0.1\%), PMA (100 nmol/l), PMA+staurosporine (10 nmol/l), TUDCA $(25 \mu \mathrm{mol} / \mathrm{l})$, and TLCA $(10 \mu \mathrm{mol} / \mathrm{l})$ for $30 \mathrm{~min}$. The results show that Mrp2 was phosphorylated under control conditions. The PMA-induced increase in phosphorylation was reversed by staurosporine. Bile acids at low micromolar concentrations did not affect total phosphorylation of Mrp2 (fig 3A).

\section{Phosphorylation of human MRP2 by $\mathrm{CPKC} \alpha, \mathrm{nPKC} \varepsilon$ and PKA}

Human MRP2 ${ }^{33}$ was immunoprecipitated from HepG2 hepatoma cells and MRP2 phosphorylation was studied in vitro using recombinant activated PKC $\alpha$, PKC $\varepsilon$ or PKA. The results show that the MRP2 complex after immunoprecipitation was phosphorylated by $\mathrm{cPKC} \alpha, \mathrm{nPKC} \varepsilon$ and $\mathrm{PKA}$ (fig $3 \mathrm{~B}$ ). Combined
Table 3 The cytoprotective effect of tauroursodeoxycholic acid in taurolithocholic acid-induced cholestasis is impaired by combined inhibition of $\mathrm{c}$ protein kinase $\mathrm{C}$ and protein kinase $\mathrm{A}$

\begin{tabular}{llll}
\hline & & LDH release & \\
\cline { 3 - 4 } Bile acid & Inhibitor & Concentration (mU/min/g liver) & $\%$ of controls \\
\hline DMSO & & $2.3(1.2)$ & 100 \\
DMSO & ST+H89 & $4.0(5.1)$ & 173 \\
TUDCA & & $11.4(4.4)$ & 100 \\
TUDCA & ST+H89 & $11.7(6.8)$ & 103 \\
TLCA & & $73.1(31.7)$ & 100 \\
TLCA & ST+H89 & $61.3(29.3)$ & 84 \\
TLCA+TUDCA & & $21.3(7.3)$ & 100 \\
TLCA+TUDCA & ST & $71.8(34.2)$ & 336 \\
TLCA+TUDCA & Gö6976 & $40.5(43.3)$ & 190 \\
TLCA+TUDCA & H89 & $39.3(7.2)$ & 184 \\
TLCA+TUDCA & ST+H89 & $95.3(24.7)^{*}$ & 446 \\
TLCA+TUDCA & Gö6976+H89 & $64.1(53.5)$ & 300 \\
TLCA+TCA & & $2.4(5.3)$ & 100 \\
TLCA+TCA & ST+H89 & $65.3(52.0)$ & 292 \\
\hline
\end{tabular}

Hepatovenous lactate dehydrogenase (LDH) release as a readout of hepatocellular damage was determined during steady state at $85 \mathrm{~min}$ in perfused rat livers after addition of the bile acids TLCA $(10 \mu \mathrm{mol} / \mathrm{l})$, TUDCA $(25 \mu \mathrm{mol} / \mathrm{l})$, cholic acid (TCA; $25 \mu \mathrm{mol} / \mathrm{l}$ ), the nonselective PKC inhibitor staurosporine (ST; $10 \mathrm{nmol} / \mathrm{I})$, the selective cPKC inhibitor Gö6976 (100 nmol/l), and/or the selective PKA inhibitor H89 (100 nmol/l) (see also fig 1). Results are given as mean (SD) of four to five experiments, each. Experiments with inhibitors are also given as \% of respective inhibitor-free controls (100\%).

${ }^{*} p<0.05$ vs respective bile acid control, ANOVA post hoc test. DMSO, dimethylsulfoxide.

incubation with $\mathrm{cPKC} \alpha$ and $\mathrm{nPKC} \varepsilon$ tended to induce a more pronounced phosphorylation than incubation with $\mathrm{cPKC} \alpha$ or $\mathrm{nPKC} \varepsilon$ alone in line with the assumption that different PKC isoforms induce phosphorylation of the MRP2 complex at different sites.

\section{DISCUSSION}

The present study demonstrates that the short-term anticholestatic effect of TUDCA in the established experimental model of TLCA-induced cholestasis is mediated mainly by a cooperative cPKC $\alpha$-/PKA-dependent mechanism.

We and others have previously observed that TUDCA at low micromolar concentrations stimulates $\mathrm{Ca}^{2+}$ entry into hepatocytes independent of inositol-1,4,5-trisphosphate, ${ }^{11-13}$ selectively translocates $\mathrm{Ca}^{2+}$-dependent $\mathrm{CPKC} \alpha$ to hepatocyte membranes, ${ }^{14} 2034$ stimulates formation of $s, n$-1,2-diacylglycerol (DAG), ${ }^{14}$ activates membrane-bound $\mathrm{PKC}^{14} 34$ and stimulates impaired biliary secretion of organic anions and bile acids in TLCA-induced cholestasis by PKC-dependent mechanisms in isolated perfused rat liver (IPRL) ${ }^{20}$ and isolated rat hepatocyte couplets, ${ }^{22}$ respectively. Similar effects of TUDCA on $\left[\mathrm{Ca}^{2+}\right]_{\mathrm{i}}$ and $\mathrm{Ca}^{2+}$-dependent $\mathrm{cPKC} \alpha$ have been observed in cholangiocytes. ${ }^{35}$ The present study not only confirms a role of $\mathrm{PKC}$, but, by use of the cPKC-specific inhibitor Gö6976 (figs 1B and 2A,B), specifically shows that a $\mathrm{Ca}^{2+}$-dependent $\mathrm{PKC}$ isoform mediates the anticholestatic effect of TUDCA. Among the known cPKC isoforms, $c P K C \alpha, c P K C \beta-\mathrm{I}$, cPKC $\beta$-II and $c \mathrm{PKC} \gamma$, only $c \mathrm{PKC} \alpha$ and $\mathrm{cPKC} \beta$-II have been detected in hepatocytes. We were not able to disclose translocation of $c P K C \beta-I I$ by TUDCA to hepatocyte membranes in IPRL, IRHC and Ntcp-transfected HepG2 hepatoma cells (data not shown; see also Beuers et a ${ }^{14}$ ). Thus, $c \mathrm{PKC} \alpha$ is most likely the PKC isoform involved in the anticholestatic action of TUDCA in experimental cholestasis as suggested previously. ${ }^{14} 2036$ 
A

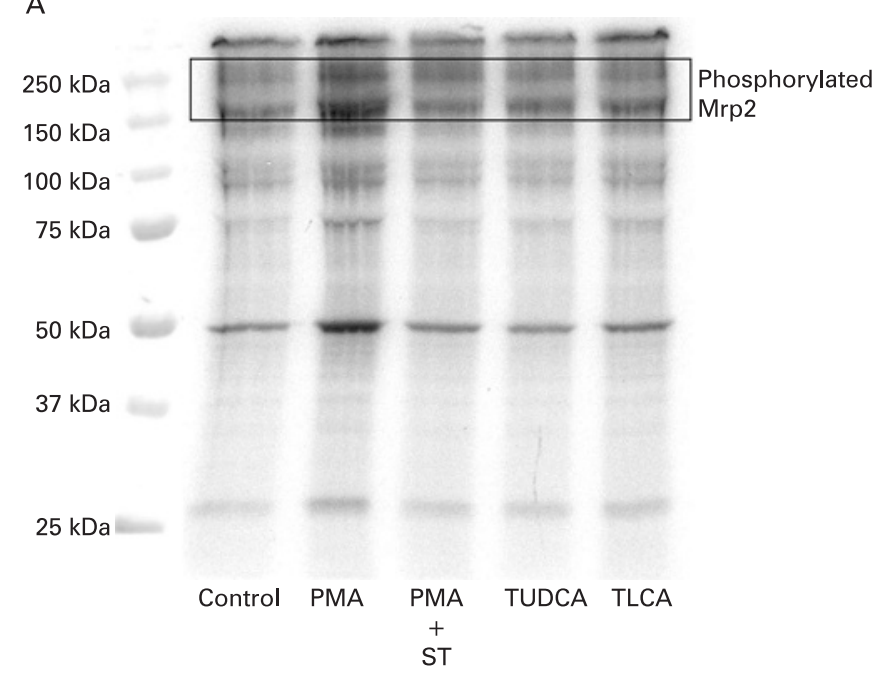

B

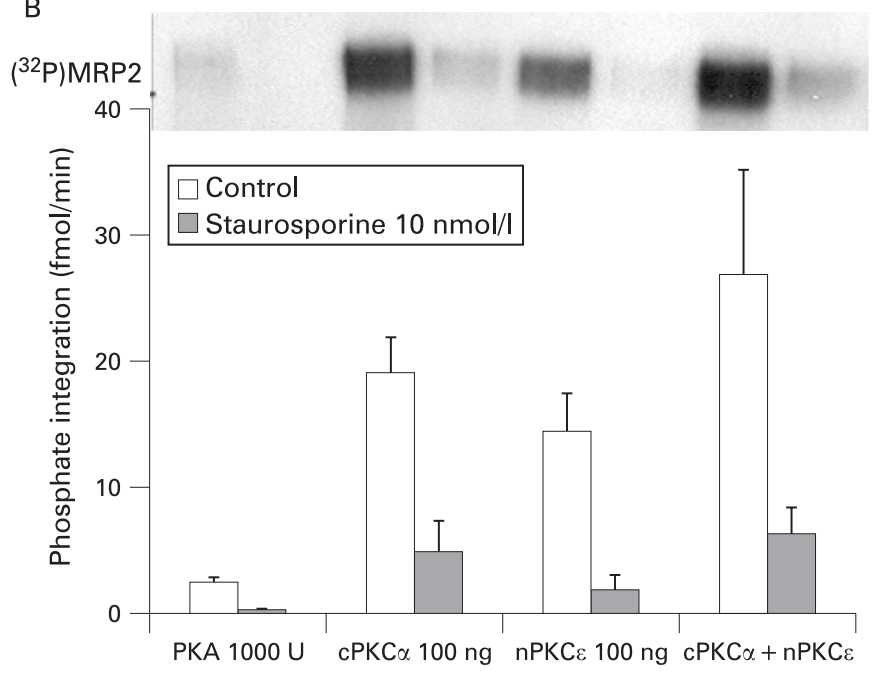

Figure 3 The conjugate export pump, Mrp2/Abcc2, is phosphorylated by protein kinase $C$ (PKC) and protein kinase $A(P K A)$ in a staurosporinesensitive way. (A) Rat hepatocyte total Mrp2/Abcc2 phosphorylation was stimulated by phorbol 12-myristate 13-acetate (PMA), in a staurosporine-sensitive way, but not by bile acids at low micromolar concentrations. Rat hepatocytes were preloaded with ${ }^{32} \mathrm{P}$ orthophosphoric acid and then treated for $4 \mathrm{~h}$ with dimethyl sulfoxide (DMSO) (0.1\%, v/v), $100 \mathrm{nmol} / \mathrm{I} \mathrm{PMA,} 100 \mathrm{nmol} / \mathrm{I} \mathrm{PMA} \mathrm{+} 10 \mathrm{nmol} / \mathrm{l}$ staurosporine, $25 \mu \mathrm{mol} / \mathrm{l}$ tauroursodeoxycholic acid (TUDCA) or $10 \mu \mathrm{mol} / \mathrm{l}$ taurolithocholic acid (TLCA). Cells were then homogenised, Mrp2/Abcc2 was immunoprecipitated and immunoblotted as described in Materials and methods. A pilot experiment is shown. (B) Human MRP2/ABCC2 was phosphorylated in vitro by recombinant $\mathrm{CPKC} \alpha$, $\mathrm{nPKC} \varepsilon$, and PKA in a staurosporine-sensitive way. MRP2/ABCC2 was immunoprecipitated from human HepG2 hepatoma cells and was exposed to recombinant $\mathrm{cPKC} \alpha, \mathrm{nPKC} \varepsilon$, and PKA for $2 \mathrm{~h}$ in the absence or presence of the potent PKC inhibitor staurosporine (ST, $10 \mathrm{nmol} / \mathrm{l}$ ). MRP2/ABCC2 phosphorylation in the presence of ${ }^{32} \mathrm{P}$-ATP was determined radiochemically as described in Materials and methods. Results are given as means (SD) of three independent preparations.

A role of $\mathrm{CPKC} \alpha$ as a mediator of the choleretic effect of TUDCA in IPRL was recently questioned and the cPKC agonist thymeleatoxin was shown to induce cholestasis in IPRL. ${ }^{37}$ In contrast to thymeleatoxin, TUDCA interacts with various hepatocellular (and cholangiocellular) signalling cascades. ${ }^{9}$ Their impact on control of diverse liver cell functions by TUDCA in health and under cholestatic conditions remains to be further unravelled. Our previous data were in line with the assumption that bile flow and organic anion secretion under normal "noncholestatic" conditions are modulated by TUDCA mostly independent of $\mathrm{Ca}^{2+}$ and $\mathrm{Ca}^{2+}$-dependent $\mathrm{cPKC} \alpha$ (fig 1C; see also Beuers et $a l^{12}$ ) whereas $\mathrm{Ca}^{2+}$-dependent $\mathrm{cPKC} \alpha$ appears to play a key role in the anticholestatic action of TUDCA in TLCA-induced cholestasis. ${ }^{20}$

A relevant effect of TUDCA on hepatocellular PKA activity has not been observed ${ }^{15}$ although PKA is a well-known mediator of apical secretion in hepatocytes. ${ }^{38}$ In line with previous findings, we were unable to observe an effect of TUDCA on cAMP levels and PKA activity as reflected by unchanged CREB phosphorylation in liver tissue. In addition, the selective PKA inhibitor H89 alone did not affect TUDCA-induced bile secretion in IPRL in the present study. It should be kept in mind, however, that global determination of CREB phosphorylation and CAMP in liver tissue may trivialise the complexity of PKA action and cAMP signals in microdomains of the apical plasma membrane. ${ }^{39}$ This assumption is strengthened by the recent finding that type II inositol-1,4,5-trisphosphate receptor (InsP3) isoforms are concentrated in the pericanalicular region in a lipid raft-dependent way. ${ }^{40}{ }^{41}$ Type II InsP3 are essential for hepatocellular $\mathrm{Ca}^{2+}$ wave formation and, possibly, $\mathrm{Ca}^{2+} / \mathrm{cPKC} \alpha /$ PKA interaction. Thus, more sophisticated methodological approaches will be needed to further elucidate the possibly permissive role of PKA in the anticholestatic action of TUDCA in the pericanalicular zone of hepatocytes. ${ }^{9}$

Under non-cholestatic conditions, an integrin-dependent dual signalling pathway involving the MAPK Erk1/2 and p38MAPK plays a role in mediating the choleretic effect of TUDCA in IPRL. ${ }^{16-19}$ We were not able to show a role of MAPK for the anticholestatic action of TUDCA in TLCA-induced cholestasis when either Erk1/2 or p38MAPK or Erk1/2 and p38MAPK concomitantly were inhibited. ${ }^{30}$ Thus, different signalling pathways mediate the choleretic and the anticholestatic action of TUDCA in hepatocytes. ${ }^{9}$

A cooperative stimulation of secretory activity by PKC/PKA as assumed in the present model for the anticholestatic action of TUDCA has previously been described in different cell types. ${ }^{28} 29$ Apical carriers and their anchoring proteins ${ }^{42}{ }^{43}$ are potential targets of a concerted action of PKA and $\mathrm{cPKC} \alpha$ at the canalicular hepatocyte membrane for TUDCA-induced carrier insertion as well as of $\mathrm{nPKC} \varepsilon$ in TLCA-induced carrier retrieval. ${ }^{9}$ The deduced amino acid sequences of the conjugate and bile salt export pumps, Mrp2/Abcc2 and Bsep/Abcb11, respectively, show numerous potential serine/threonine phosphorylation sites for PKC and PKA. We found that Mrp2/Abcc2 can be phosphorylated in vitro in rat hepatocytes by PKC agonists or after immunoprecipitation from HepG2 hepatoma cells by recombinant activated $\mathrm{cPKC} \alpha, \mathrm{nPKC} \varepsilon$, and $\mathrm{PKA}$. Interestingly, Mrp2/Abcc2 transport activity has recently been shown to be stimulated by $\mathrm{cPKC} \alpha$ in a baculovirus coexpression system in vitro. ${ }^{44}$ These findings are in line with those previously observed for Bsep/Abcb11 ${ }^{31}$ which also can be phosphorylated by cPKC $\alpha$. Identification of key serine/threonine phosphorylation sites required for apical insertion and retrieval of $\mathrm{Mrp2} / \mathrm{Abcc} 2$ or BSEP/ABCB11 - as demonstrated for the sodium taurocholate cotransporting polypeptide, Ntcp/Slc10a1, in the basolateral membrane ${ }^{45}$ - will be a prerequisite to further unravel the cholestatic and anticholestatic post-translational effects of bile acids in hepatocytes.

In conclusion, the well-known anticholestatic effect of TUDCA is largely blocked by selective pharmacological intervention in the present study, indicating that TUDCA exerts its 
post-translational anticholestatic effect mainly by a cooperative cPKC $\alpha$-/PKA-dependent mechanism in the experimental model of TLCA-induced cholestasis.

Funding: This work was supported by a grant from the Deutsche Forschungsgemeinschaft (DFG Be 1242/5-5).

Competing interests: None.

\section{REFERENCES}

1. Poupon RE, Lindor KD, Cauch-Dudek K, et al. Combined analysis of randomized controlled trials of ursodeoxycholic acid in primary biliary cirrhosis. Gastroenterology 1997:113:884-90.

2. Paumgartner G, Beuers U. Mechanisms of action and therapeutic efficacy of ursodeoxycholic acid in cholestatic liver disease. Clin Liver Dis 2004;8:67-81, vi.

3. Corpechot C, Carrat F, Bonnand AM, et al. The effect of ursodeoxycholic acid therapy on liver fibrosis progression in primary biliary cirrhosis. Hepatology 2000:32:1196-9.

4. Pares A, Caballeria L, Rodes J, et al. Long-term effects of ursodeoxycholic acid in primary biliary cirrhosis: results of a double-blind controlled multicentric trial. UDCACooperative Group from the Spanish Association for the Study of the Liver. J Hepatol 2000;32:561-6.

5. Lindor KD, Jorgensen RA, Therneau TM, et al. Ursodeoxycholic acid delays the onset of esophageal varices in primary biliary cirrhosis. Mayo Clin Proc 1997;72:1137-40.

6. Corpechot C, Carrat F, Bahr A, et al. The effect of ursodeoxycholic acid therapy on the natural course of primary biliary cirrhosis. Gastroenterology 2005;128:297-303.

7. ter Borg PC, Schalm SW, Hansen BE, et al. Prognosis of ursodeoxycholic acidtreated patients with primary biliary cirrhosis. Results of a 10-yr cohort study involving 297 patients. Am J Gastroenterol 2006;101:2044-50.

8. Pares A, Caballeria L, Rodes J. Excellent long-term survival in patients with primary biliary cirrhosis and biochemical response to ursodeoxycholic acid. Gastroenterology 2006;130:715-20.

9. Beuers U. Drug insight: Mechanisms and sites of action of ursodeoxycholic acid in cholestasis. Nature Clin Prac 2006;3:318-28.

10. Anwer MS. Cellular regulation of hepatic bile acid transport in health and cholestasis. Hepatology 2004;39:581-90.

11. Beuers U, Nathanson MH, Boyer JL. Effects of tauroursodeoxycholic acid on cytosolic $\mathrm{Ca}^{2+}$ signals in isolated rat hepatocytes. Gastroenterology 1993;104:604-12.

12. Beuers U, Nathanson MH, Isales CM, et al. Tauroursodeoxycholic acid stimulates hepatocellular exocytosis and mobilizes extracellular $\mathrm{Ca}^{++}$mechanisms defective in cholestasis. J Clin Invest 1993;92:2984-93.

13. Bouscarel B, Fromm H, Nussbaum R. Ursodeoxycholate mobilizes intracellular $\mathrm{Ca}^{2+}$ and activates phosphorylase a in isolated hepatocytes. Am J Physiol 1993;264 (2 Pt 1):G243-51.

14. Beuers U, Throckmorton DC, Anderson MS, et al. Tauroursodeoxycholic acid activates protein kinase $\mathrm{C}$ in isolated rat hepatocytes. Gastroenterology 1996;110:1553-63

15. Bouscarel B, Kroll SD, Fromm H. Signal transduction and hepatocellular bile acid transport: cross talk between bile acids and second messengers. Gastroenterology 1999;117:433-52.

16. Schliess F, Kurz AK, vom Dahl S, et al. Mitogen-activated protein kinases mediate the stimulation of bile acid secretion by tauroursodeoxycholate in rat liver. Gastroenterology 1997;113:1306-14.

17. Kurz AK, Graf D, Schmitt M, et al. Tauroursodesoxycholate-induced choleresis involves p38 (MAPK) activation and translocation of the bile salt export pump in rats. Gastroenterology 2001:121:407-19.

18. Haussinger D, Kurz AK, Wettstein $\mathbf{M}$, et al. Involvement of integrins and Src in tauroursodeoxycholate-induced and swelling-induced choleresis. Gastroenterology 2003;124:1476-87.

19. Kubitz R, Sutfels G, Kuhlkamp T, et al. Trafficking of the bile salt export pump from the Golgi to the canalicular membrane is regulated by the p38 MAP kinase. Gastroenterology 2004;126:541-53.

20. Beuers U, Bilzer M, Chittattu A, et al. Tauroursodeoxycholic acid inserts the apical conjugate export pump, Mrp2, into canalicular membranes and stimulates organic anion secretion by protein kinase $\mathrm{C}$-dependent mechanisms in cholestatic rat liver. Hepatology 2001;33:1206-16.

21. Dombrowski F, Stieger B, Beuers U. Tauroursodeoxycholic acid inserts the bile salt export pump into canalicular membranes of cholestatic rat liver. Lab Invest 2006;86:166-74.

22. Milkiewicz $\mathbf{P}$, Roma MG, Elias E, et al. Hepatoprotection with tauroursodeoxycholate and beta muricholate against taurolithocholate induced cholestasis: involvement of signal transduction pathways. Gut 2002;51:113-9.

23. Javitt J. Cholestasis in rats induced by taurolithocholate. Nature 1966;210:1262-63.

24. Beuers U, Denk GU, Soroka CJ, et al. Taurolithocholic acid exerts cholestatic effects via phosphatidylinositol 3-kinase-dependent mechanisms in perfused rat livers and rat hepatocyte couplets. J Biol Chem 2003;278:17810-8.

25. Anwer MS, Engelking LR, Nolan K, et al. Hepatotoxic bile acids increase cytosolic $\mathrm{Ca}^{++}$activity of isolated rat hepatocytes. Hepatology 1988;8:887-91.

26. Beuers U, Probst I, Soroka C, et al. Modulation of protein kinase $\mathrm{C}$ by taurolithocholic acid in isolated rat hepatocytes. Hepatology 1999;29:477-82.

27. Bouscarel B, Gettys TW, Fromm H, et al. Ursodeoxycholic acid inhibits glucagoninduced cAMP formation in hamster hepatocytes: a role for PKC. Am J Physiol 1995;268 (2 Pt 1):G300-10.

28. Chen Y, Button B, Altenberg GA, et al. Potentiation of effect of PKA stimulation of Xenopus CFTR by activation of PKC: role of NBD2. Am J Physiol Cell Physiol 2004;287:C1436-44.

29. Wan OF, Dong Y, Yang H, et al. Protein kinase activation increases insulin secretion by sensitizing the secretory machinery to $\mathrm{Ca}^{2+}$. J Gen Physiol 2004;124:653-62.

30. Denk GU, Hohenester S, Wimmer R, et al. Role of mitogen-activated protein kinases in tauroursodeoxycholic acid-induced bile formation in cholestatic rat liver. Hepatol Res 2008;38:717-26.

31. Noe J, Hagenbuch B, Meier PJ, et al. Characterization of the mouse bile salt export pump overexpressed in the baculovirus system. Hepatology 2001;33:1223-31.

32. Miyai K, Richardson AL, Mayr W, et al. Subcellular pathology of rat liver in cholestasis and choleresis induced by bile salts. 1. Effects of lithocholic, 3betahydroxy-5-cholenoic, cholic, and dehydrocholic acids. Lab Invest 1977;36:249-58.

33. Nies AT, Keppler D. The apical conjugate efflux pump ABCC2 (MRP2). Pflugers Arch 2007:453:643-59

34. Stravitz RT, Rao YP, Vlahcevic ZR, et al. Hepatocellular protein kinase $\mathrm{C}$ activation by bile acids: implications for regulation of cholesterol 7 alpha-hydroxylase. Am J Physiol 1996;271 (2 Pt 1):G293-303.

35. Alpini G, Baiocchi L, Glaser S, et al. Ursodeoxycholate and tauroursodeoxycholate inhibit cholangiocyte growth and secretion of BDL rats through activation of PKC alpha. Hepatology 2002;35:1041-52.

36. Baiocchi L, Tisone G, Russo MA, et al. TUDCA prevents cholestasis and canalicular damage induced by ischemia-reperfusion injury in the rat, modulating PKCalpha-ezrin pathway. Transpl Int 2008;21:792-800.

37. Kubitz R, Saha N, Kuhlkamp T, et al. $\mathrm{Ca}^{2+}$-dependent protein kinase $\mathrm{C}$ isoforms induce cholestasis in rat liver. J Biol Chem 2004;279:10323-30.

38. Benedetti A, Strazzabosco M, Ng OC, et al. Regulation of activity and apical targeting of the $\mathrm{Cl}^{-} / \mathrm{HCO}_{3}{ }^{-}$exchanger in rat hepatocytes. Proc Natl Acad Sci U S A 1994; $91: 792-6$

39. Willoughby D, Cooper DM. Organization and $\mathrm{Ca}^{2+}$ regulation of adenylyl cyclases in cAMP microdomains. Physiol Rev 2007;87:965-1010.

40. Hernandez $\mathbf{E}$, Leite MF, Guerra MT, et al. The spatial distribution of inositol 1,4,5trisphosphate receptor isoforms shapes $\mathrm{Ca}^{2+}$ waves. J Biol Chem 2007;282:10057-67.

41. Nagata J, Guerra MT, Shugrue CA, et al. Lipid rafts establish calcium waves in hepatocytes. Gastroenterology 2007;133:256-67.

42. Kikuchi S, Hata M, Fukumoto K, et al. Radixin deficiency causes conjugated hyperbilirubinemia with loss of Mrp2 from bile canalicular membranes. Nat Genet 2002;31:320-5.

43. Wang W, Soroka CJ, Mennone A, et al. Radixin is required to maintain apical canalicular membrane structure and function in rat hepatocytes. Gastroenterology 2006;131:878-84.

44. Ito K, Wakabayashi T, Horie T. Mrp2/Abcc2 transport activity is stimulated by protein kinase Calpha in a baculo virus co-expression system. Life Sci 2005;77:539-50.

45. Anwer MS, Gillin H, Mukhopadhyay S, et al. Dephosphorylation of Ser-226 facilitates plasma membrane retention of Ntcp. J Biol Chem 2005;280:33687-92. 


\section{GUT}

\section{Tauroursodeoxycholic acid exerts anticholestatic effects by a cooperative cPKC $\alpha$-/PKA-dependent mechanism in rat liver}

R Wimmer, S Hohenester, T Pusl, et al.

Gut 2008 57: 1448-1454 originally published online June 26, 2008 doi: $10.1136 /$ gut.2007.140871

Updated information and services can be found at:

http://gut.bmj.com/content/57/10/1448.full.html

\section{These include:}

References This article cites 45 articles, 9 of which can be accessed free at: http://gut.bmj.com/content/57/10/1448.full.html\#ref-list-1

Article cited in:

http://gut.bmj.com/content/57/10/1448.full.html\#related-urls

Email alerting Receive free email alerts when new articles cite this article. Sign up in service the box at the top right corner of the online article.

Topic Articles on similar topics can be found in the following collections Collections

Notes

To request permissions go to:

http://group.bmj.com/group/rights-licensing/permissions

To order reprints go to:

http://journals.bmj.com/cgi/reprintform

To subscribe to BMJ go to:

http://group.bmj.com/subscribe/ 